\title{
Sparse representation discretization errors in multi-sensor radar target motion estimation
}

\author{
Hossein Azodi, Uwe Siart, and Thomas F. Eibert \\ Chair of High-Frequency Engineering, Department of Electrical and Computer Engineering, Technical University of Munich, \\ 80290 Munich, Germany \\ Correspondence to: Hossein Azodi (hossein.azodi@tum.de)
}

Received: 27 December 2016 - Accepted: 5 August 2017 - Published: 21 September 2017

\begin{abstract}
In a multi-sensor radar for the estimation of the targets motion states, more than one module of transmitter and receiver are utilized to estimate the positions and velocities of targets, also known as motion states. By applying the compressed sensing (CS) reconstruction algorithms, the surveillance space needs to be discretized. The effect of the additive errors due to the discretization are studied in this paper. The errors are considered as an additive noise in the wellknown under-determined CS problem. By employing properties of these errors, analytical models for its average and variance are derived. Numerous simulations are carried out to verify the analytical model empirically. Furthermore, the probability density functions of discretization errors are estimated. The analytical model is useful for the optimization of the performance, the efficiency and the success rate in CS reconstruction for radar as well as many other applications.
\end{abstract}

\section{Introduction}

A multi-sensor radar system contains more than one module of transmitter and receiver. These modules are usually positioned separately to increase the spatial diversity, the coverage or both. A multi-sensor radar system might be configured in mono-static, bi-static or multi-static, also known as multiple input mutiple output (MIMO) radar (Skolnik, 1962). The estimation of the positions and velocities of moving targets, also known as motion states, in the surveillance space is a typical use-case of these radars. A more particular application of these radars is to prevent collisions among objects in the surveillance space by estimating the motion states in relatively short intervals (Azodi et al., 2013, 2015). For the data fusion and the processing, there are various possi- bilities, especially among the algorithms in the framework of compressed sensing (CS). Compressed sensing is understood as a set of information and estimation algorithms for compressible signal processing (Candes et al., 2006; Candès, 2006; Donoho, 2006; Baraniuk, 2007). Despite the ShannonNyquist theorem, which asserts a high sampling rate, CS estimation algorithms reconstruct a signal from far fewer samples. CS algorithms have been applied to multi-sensor radar systems, medical imaging, remote sensing, and many other signal recovery problems in recent years (Duarte and Eldar, 2011).

Sparse representation and finding the solution of the sparse vector $s$ in the under-determined problem

$\boldsymbol{d}=\Psi_{s}$

are the primary objectives of CS signal recovery algorithms. In Eq. (1), $\boldsymbol{\Psi}$ and $\boldsymbol{d}$ are the so-called sensing matrix and the measurement vector, respectively. Basically, Eq. (1) represents an under-determined set of linear equations, where contributions of noise and other errors are neglected. In realistic data processing, the measured vector is slightly perturbed due to unavoidable measurement system noise and modeling errors, e.g., due to truncation, rounding, or discretization. The perturbation in such cases is often modeled as a Gaussian distributed random signal. Therefore, the problem in Eq. (1) turns into

$\boldsymbol{d}=\boldsymbol{\Psi}+\boldsymbol{e}$,

where $\boldsymbol{e}$ represents the additive random signal. Modified versions of the CS reconstruction algorithms, e.g., basis pursuit de-noising (BPDN), are capable of solving the noisy problem in Eq. (2) as well (Chen et al., 2001). 
The recovery of the sparse vector from the CS underdetermined systems is successful if a number of conditions are met. Since any under-determined problem exhibits an infinite number of solutions, these conditions are necessary to make sure that the reconstructed solution is the correct one. One of the prevailing conditions is sparsity of the unknown vector $s$. Other conditions postulate certain properties of the sensing matrix $\boldsymbol{\Psi}$ like coherence, restricted isometry property (RIP), or the null-space property (NSP). These conditions and their interrelation with the sparsity of $s$ are well elaborated in Foucart and Rauhut (2013), Eldar and Kutyniok (2012) and Donoho (2006).

Recently, CS algorithms have also been utilized for the detection of point-like targets in multi-sensor or multiple-input multiple-output (MIMO) radar systems, where a sufficient number of transmitters and receivers observe the surveillance space (Eldar et al., 2010; Ender, 2013; Anitori et al., 2013). Typically in these radar systems, number and motion states, initial positions and velocities, of the targets are the unknowns. Compared with conventional methods, CS may achieve identical detection performance with a reduced number of sensors. It may also provide higher resolution and accuracy (Donoho and Tanner, 2010; Herman and Strohmer, 2009; Lehmann et al., 2006). Nevertheless, a few additional assumptions are required so that such problems become compatible with the CS recovery methods. Apart from the already mentioned sparsity of the scene, these are exact phase synchronization between transceivers, electromagnetic far-field condition, non-relativistic movements, constant radar cross section (RCS) during one observation and considering targets as point-like scatterers with their motion states at the centers of the grid cells. These assumptions, except the last one, are mostly valid for high-frequency radar systems, particularly for target motion estimation where indeed only few targets exist in the entire vacant surveillance space (Fishler et al., 2006; Haimovich et al., 2008). Also, the approximation of extended targets by an ensemble of point-like scatterers is often possible in the high-frequency regime (Hurst and Mittra, 1987).

However, a rather fine grid might be required to warrant the last assumption. Otherwise, it is not justified to assume that the motion states are located at the centers of grid cells. A very fine grid results in a highly coherent sensing matrix which not only causes more computational complexity, but also increases the ambiguity in the reconstruction process. In radar applications, such as collision avoidance radars (Azodi et al., 2014; Wächter et al., 2014), assuming a very fine discretization is not even practical as out-bound targets ${ }^{1}$ considerably enlarge the solution domain. Targets, whose true motion states lie offside the grid points of the discretized solution domain, are commonly referred to as off-grid targets

\footnotetext{
${ }^{1}$ Assuming a boundary in the scene which encompasses the radar sensors, the out-bound targets are those which are located outside this boundary.
}

(Tan and Nehorai, 2014; Nielsen et al., 2012; Tang et al., 2012; Gurbuz et al., 2013). It is beneficial to analyze the impact of off-grid targets on the CS recovery process and to modify it, accordingly. This impact is studied by analytically modeling it as errors added to the principal CS problem (1) in this work. The main purpose is to find an implicit and parameterized model of these errors. Since such errors are not limited to off-grid targets of radar applications and are seen in various CS problems, the term "discretization error" is used in this work as it reflects the source of errors in a more general and universal way than off-grid targets effect.

The majority of previous works do not render a rigorous distinction between system noise and discretization errors. Both are summarized in a single Gaussian random signal model at the right-hand side of Eq. (2), although they have different sources and might have different impacts on the problem. This work discriminates between these perturbation sources and studies the particular impact of discretization errors on CS reconstruction algorithms. In this view, the realistic CS problem is represented by

$\boldsymbol{d}=\boldsymbol{\Psi}+\boldsymbol{e}_{\mathrm{s}}+\boldsymbol{e}_{\mathrm{d}}$

where $\boldsymbol{e}_{\mathrm{s}}$ and $\boldsymbol{e}_{\mathrm{d}}$ are system noise and discretization errors, respectively. Also, Eq. (3) implies that, even in a fictitious noise-free system, where measurements are carried out with unlimited accuracy, the discretization errors $\boldsymbol{e}_{\mathrm{d}}$ still remains and cannot be ignored.

The formation of the CS under-determined problem is explained in Sect. 2. An analytical model of the received echo is considered based on geometrical optics. Utilizing the signal model in Sect. 2, the approximated discretization errors are calculated in Sect. 3 for two-dimensional geometries. The methods and models of this work are not limited to two-dimensional problems and can be extended to threedimensional configurations. The proposed model is tested by and verified against an extensive number of computer simulations. The results of these numerical experiments are gathered in Sect. 4. Section 5 finalizes the paper with some conclusion remarks.

\section{Single carrier continuous wave signal model}

A typical 2-D scene for target motion estimation using radar sensors is illustrated in Fig. 1. A small number of targets (marked by stars) move in the surveillance area of a Doppler radar system with $N$ mono-static sensors. The wavelength of the transmitted signal as compared to the physical dimensions is considered short enough to apply the asymptotic approximations in the calculation of the received signal and to model it analytically.

In a single frequency continuous wave (CW) radar system, each sensor transmits the signal

$w_{n}(t)=\exp \left\{j 2 \pi f_{\mathrm{c}} t+\theta_{n}\right\}$, 
where $f_{\mathrm{c}}$ and $\theta_{n}$ are the carrier frequency and initial phase of the transmitted signal. The received echo from the targets at the $n$th sensor is

$d_{n}(t)=\sum_{p=1}^{P} a_{p} \exp \left\{j 2 \pi f_{\mathrm{c}}\left(t-\tau_{p, n}\right)+\theta_{n}\right\}+e_{\mathrm{S}}(t)$,

where $e_{\mathrm{S}}(t)$ is a random signal with zero-mean Gaussian distribution $\mathcal{N}(0, \sigma)$ to represent the system noise. Each coefficient $a_{p}$ represents the amplitude of the echo received from the $p$ th target. These include the system response, free space path loss, and the target's RCS. Also, $\tau_{p, n}$ is the effective round-trip delay between the $n$th module and the $p$ th target. Having the $n$th module at $\boldsymbol{r}_{n}=r_{x n} \hat{x}+r_{y n} \hat{y}$ and the $p$ th target at

$\boldsymbol{r}_{p}=\left(r_{x p}+v_{x p} t\right) \hat{x}+\left(r_{y p}+v_{y p} t\right) \hat{y}$,

the round-trip delay is

$\tau_{p, n}=\frac{2}{c_{0}}\left(\left(r_{x p}-r_{x n}+v_{x p} t\right)^{2}+\left(r_{y p}-r_{y n}+v_{y p} t\right)^{2}\right)^{\frac{1}{2}}$.

The carrier $\exp \left\{j 2 \pi f_{\mathrm{c}} t+\theta_{n}\right\}$ in Eq. (5) is removed after down-conversion and subsequent analog-to-digital conversion (ADC). Sampling generates the data series

$d_{n}[i]=\sum_{p=1}^{P} a_{p} \chi_{p, n}[i]+\widetilde{e}_{\mathrm{S}}[i]$,

where $\widetilde{e}_{\mathrm{s}}(t)$ is the colored baseband noise and

$$
\begin{aligned}
\chi_{p, n}[i] & =\exp \left\{-j 4 \pi \frac{f_{\mathrm{c}}}{c_{0}}\left(\left(r_{x p}-r_{x n}+v_{x p} i T\right)^{2}\right.\right. \\
& \left.\left.+\left(r_{y p}-r_{y n}+v_{y p} i T\right)^{2}\right)^{\frac{1}{2}}\right\}
\end{aligned}
$$

where $c_{0}$ is the vacuum speed of light and $T$ is the sampling interval.

Let's assume that every sensor collects $L$ samples during one observation. The total number of $N \times L$ independent data samples is concatenated in a column vector $\boldsymbol{d}$ according to

$\boldsymbol{d}=\left[\begin{array}{c}d_{1}[1], \ldots, d_{1}[L], \\ d_{2}[1], \ldots, d_{2}[L] \\ \vdots \\ d_{N}[1], \ldots, d_{N}[L],\end{array}\right]^{\mathrm{T}}$

where the superscript $\mathrm{T}$ denotes the vector transpose. By maintaining the concatenation order in Eq. (10), Eq. (8) is rewritten in matrix form according to

$\boldsymbol{d}=\boldsymbol{X} \boldsymbol{a}+\widetilde{\boldsymbol{e}}_{\mathrm{s}}$.

Vector $\widetilde{\boldsymbol{e}}_{\mathrm{S}}$ embodies the additive noise in each sample. The amplitudes of the echoes are gathered in $\boldsymbol{a}=\left[a_{1} \ldots a_{P}\right]^{\mathrm{T}}$ and

$\boldsymbol{X}=\left[\begin{array}{cccc}\chi_{1,1}[1] & \chi_{2,1}[1] & \ldots & \chi_{P, 1}[1] \\ \chi_{1,1}[2] & \chi_{2,1}[2] & \ldots & \chi_{P, 1}[2] \\ \vdots & \vdots & \ldots & \vdots \\ \chi_{1, N}[L] & \chi_{2, N}[L] & \ldots & \chi_{P, N}[L]\end{array}\right]$.

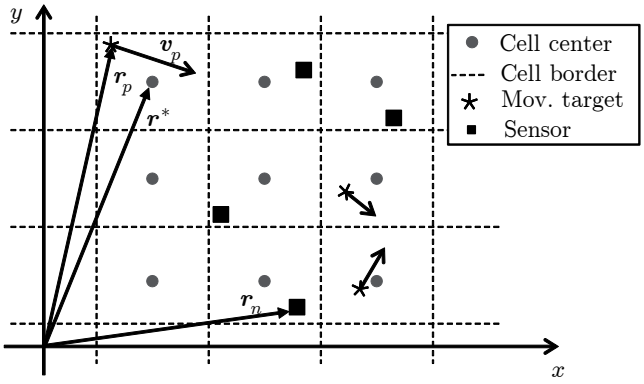

Figure 1. Targets move in the surrounding space of a multi-sensor radar system. The sensors and the targets are displayed by squares and stars, respectively. The arrows illustrate relative velocities of the targets. The dashed lines are the border of the cells. The centers of the cells are depicted by circles.

Every column of $\boldsymbol{X}$ represents the received samples of a target following the same concatenation order as $\boldsymbol{d}$. For example, in explicit notation, the $p$ th column of $\boldsymbol{X}$ is

$\chi_{p}=\left[\begin{array}{c}\chi_{p, 1}[1], \ldots, \chi_{p, 1}[L], \\ \chi_{p, 2}[1], \ldots, \chi_{p, 2}[L], \\ \vdots \\ \chi_{p, N}[1], \ldots, \chi_{p, N}[L]\end{array}\right]^{\mathrm{T}}$.

Equation (11) is not yet in a suitable form, since the unknowns appear in both $\boldsymbol{X}$ and $\boldsymbol{a}$. Furthermore, the size of $\boldsymbol{a}$ is unknown as usually in a radar problem a priori knowledge about the number of targets is not available. Hence, a systematic solution algorithm cannot be proposed yet.

This is, where the CS methodology comes into play. It transforms Eq. (11) into a solvable set of equations in the form

$d=Z s+e$

Each column of $\boldsymbol{Z}$ corresponds to one element from the discretized solution domain $\mathbb{S}_{\mathrm{d}}$. The solution domain is a set of all considered target positions together with all permissible velocities, also referred to as states. The matrix $\boldsymbol{Z}$ has, therefore, an extremely large number of columns as it includes one column for each possible state. The vector $s$ is the unknown solution vector of Eq. (13). It maps the matrix $\boldsymbol{Z}$ to the problem in Eq. (11), i.e., the non-zero elements of $s$ correspond to the occupied states found in the measured data.

If, in Eq. (12), one perceives $\chi_{p}$ as a function of $r_{x p}, r_{y p}, v_{x p}$ and $v_{y p}$ in the form $\chi_{p}=\chi\left(r_{x p}, r_{y p}, v_{x p}, v_{y p}\right)$, then the $q$ th column of $\boldsymbol{Z}$ is

$\zeta_{q}=\chi\left(r_{x q}, r_{y q}, v_{x q}, v_{y q}\right)$

where $r_{x q}, r_{y q}, v_{x q}$ and $v_{y q}$ are just the $q$ th state tuple.

Assuming that the particular detection and surveillance problem allows for limited ranges of space and velocity, the grid of the discretized solution domain is limited in extent. 
This a priori knowledge is relevant for arranging and solving Eq. (13). Short range radars and radar networks with in-bound targets can be cited as exemplary applications. In fact, the search for motion states within an unlimited variety may become highly ambiguous. With limited ranges the discretized solution domain is a set of the form

$$
\begin{aligned}
\mathbb{S}_{\mathrm{d}} & =\left\{\left(r_{x q}, r_{y q}, v_{x q}, v_{y q}\right) \mid-R \leq r_{x q}, r_{y q} \leq R,\right. \\
& \left.-V \leq v_{x q}, v_{y q} \leq V, q=1,2, \ldots, Q\right\} .
\end{aligned}
$$

Notice that the elements of the solution domain belong to $\mathbb{R}^{4}$ since they cover 2-D locations together with 2-D velocities. With a sufficiently fine discretization of the solution domain, the motion states of the targets are estimated by

$s_{q}= \begin{cases}a_{p} & \text { if } p \text { th target is at } q \text { th bin, } \\ 0 & \text { otherwise. }\end{cases}$

For the rest of this work, it is assumed that $a_{p}$ is equal to one for all targets and radar constellations. If only $P$ targets exist in the scene, $s$ is a $P$-sparse vector with the nonzeros corresponding to the amplitudes of the existing targets. Thus, Eq. (13) represents a CS problem based on the assumption that $P \ll Q$ and $N L<Q$. The vector $s$ maps the sensing matrix $\boldsymbol{Z}$ to the measured data. By definition, it is zero everywhere except for the corresponding elements due to the existing targets. Therefore, the term $\boldsymbol{Z} \boldsymbol{s}$ in Eq. (13) is the weighted summation of the selected $P$ columns of $\boldsymbol{Z}$ corresponding to the non-zeros of $\boldsymbol{s}$.

In Eq. (13), $\boldsymbol{e}$ represents both, system noise and discretization errors. For the rest of this work, the system noise is ignored. Discretization errors as the remaining part of $\boldsymbol{e}$ are introduced and studied in the next section. System noise may be added to the proposed model as a Gaussian distributed random variable for any future analysis.

\section{Discretization errors model}

As the target states may lay aside from the centers of the grid cells, the received signal $\boldsymbol{d}$ is not necessarily equal to the summation of the corresponding $P$ columns in $\boldsymbol{Z}$. As a result, the CS reconstructed signal of a successful recovery is not exactly equal to the measured echoes. This inevitable difference exists even though the discretization is very fine. The characterization of this error plays an important role for the implementation of an efficient and robust recovery algorithm. As the error is the result of modeling, improving system parameters such as the antenna or the amplifier gains do not help to suppress it. An extremely fine mesh may reduce the discretization errors but only at the cost of a more complex recovery process. Therefore, the better solution is to specifically characterize this error and to modify the recovery algorithm accordingly.

For the characterization of discretization errors, it is assumed that the vector $\boldsymbol{d}$ is measured with unlimited accuracy and the corresponding solution of the CS problem, i.e., the optimum solution, is known. The discretization errors are the difference between the optimum solution $s^{\text {opt }}$ and the received signal as

$e_{\mathrm{d}}=\boldsymbol{d}-\boldsymbol{Z} \boldsymbol{s}^{\mathrm{opt}}$.

At this point, it is also assumed that there is only one target present in the scene. As Eq. (17) is linear and existence, positions and velocities of the targets are independent, the discretization errors of more than one target are simply the summation of the discretization errors as in

$\boldsymbol{e}_{\mathrm{d}}=\sum_{p=1}^{P} \boldsymbol{e}_{\mathrm{d} p}$,

where $\boldsymbol{e}_{\mathrm{d} p}$ is the discretization errors due to the $p$ th target. It is expected that the optimum solution $\boldsymbol{s}^{\mathrm{opt}}$ corresponds to the closest $\left(r_{x}^{\mathrm{opt}}, r_{y}^{\mathrm{opt}}, v_{x}^{\mathrm{opt}}, v_{y}^{\mathrm{opt}}\right)$ in the discretized solution domain to the target true position and velocity $\left(r_{x}^{\mathrm{tar}}, r_{y}^{\mathrm{tar}}, v_{x}^{\mathrm{tar}}, v_{y}^{\mathrm{tar}}\right)$, which means

$$
\begin{aligned}
\boldsymbol{r}^{\mathrm{tar}} & =\boldsymbol{r}^{\mathrm{opt}}+\Delta \boldsymbol{r} \\
& =\left(r_{x}^{\mathrm{opt}}+v_{x}^{\mathrm{opt}} t+u_{1} \Delta r_{x} / 2+u_{3} \Delta v_{x} t / 2\right) \hat{x} \\
& +\left(r_{y}^{\mathrm{opt}}+v_{y}^{\mathrm{opt} t} u_{2} \Delta r_{y} / 2+u_{4} \Delta v_{y} t / 2\right) \hat{y}
\end{aligned}
$$

where $\Delta r_{x}, \Delta r_{y}, \Delta v_{x}$ and $\Delta v_{y}$ are discretization steps in $r_{x}, r_{y}, v_{x}$ and $v_{y}$, respectively, and $u_{m}$ for $i=1,2,3,4$ are random variables with uniform distributions

$f_{U_{m}}\left(u_{m}\right)= \begin{cases}1 / 2 & -1 \leq u_{m} \leq 1 \\ 0 & \text { otherwise }\end{cases}$

because every position and every velocity are assumed equally likely. As no preference for the position of the sensors is considered, the characteristics of the discretization errors such as expected value or variance becomes independent of the sensors. It is, therefore, sufficient to study the discretization errors in a one-sensor scene. Under these circumstances, the discretization errors term turns into

$e_{\mathrm{d}}[i]=\exp \left\{-j 2 \pi f_{\mathrm{c}} \tau^{\mathrm{tar}}\right\}-\exp \left\{-j 2 \pi f_{\mathrm{c}} \tau^{\mathrm{opt}}\right\}$.

Assuming $\Delta \tau=\tau^{\mathrm{tar}}-\tau^{\mathrm{opt}}$ is the difference between the true round-trip time of the target and its estimate, $e_{\mathrm{d}}[i]$ is approximated as

$e_{\mathrm{d}}[i]=-j 2 \pi f_{\mathrm{c}} \Delta \tau \exp \left\{-j 2 \pi f_{\mathrm{c}} \tau^{\mathrm{opt}}\right\}+\mathcal{O}\left(\Delta \tau^{2}\right)$

based on the Taylor expansion up to the first order and that $\Delta \tau \ll \tau^{\text {tar }}$. Using a Taylor expansion to approximate $\Delta \boldsymbol{r}$, it is found that,

$$
\begin{aligned}
\left|\boldsymbol{r}^{\mathrm{opt}}+\Delta \boldsymbol{r}-\boldsymbol{r}^{\mathrm{sen}}\right| & =\left|\boldsymbol{r}^{\mathrm{opt}}-\boldsymbol{r}^{\mathrm{sen}}\right|+\frac{\left(\boldsymbol{r}^{\mathrm{opt}}-\boldsymbol{r}^{\mathrm{sen}}\right) \cdot \Delta \boldsymbol{r}}{\left|\boldsymbol{r}^{\mathrm{opt}}-\boldsymbol{r}^{\mathrm{sen}}\right|} \\
& +\mathcal{O}\left(|\Delta \boldsymbol{r}|^{2}\right),
\end{aligned}
$$

where $\boldsymbol{r}^{\text {sen }}$ indicates the position of the sensor in the scene. Hence,

$\Delta \tau \approx \frac{2}{c} \frac{\left(\boldsymbol{r}^{\mathrm{opt}}-\boldsymbol{r}^{\mathrm{sen}}\right) \cdot \Delta \boldsymbol{r}}{\left|\boldsymbol{r}^{\mathrm{opt}}-\boldsymbol{r}^{\mathrm{sen}}\right|}$. 
Using the approximated differences, $e_{\mathrm{d}}[i]$ becomes

$$
\begin{array}{r}
e_{\mathrm{d}} \approx-j \frac{4 \pi f_{\mathrm{c}}}{c_{0}} \frac{\left(\boldsymbol{r}^{\mathrm{opt}}-\boldsymbol{r}^{\mathrm{sen}}\right) \cdot \Delta \boldsymbol{r}}{\left|\boldsymbol{r}^{\mathrm{opt}}-\boldsymbol{r}^{\mathrm{sen}}\right|} \\
\exp \left\{-j \frac{4 \pi f_{\mathrm{c}}}{c_{0}}\left|\boldsymbol{r}^{\mathrm{opt}}-\boldsymbol{r}^{\mathrm{sen}}\right|\right\} .
\end{array}
$$

This linear approximation is accurate as long as the discretization steps in both, space and velocity, are small enough. However, in a typical Doppler-only configuration, the size of the discretization steps in position can be even larger than the wavelength. In these configurations, the initial phase error due to the position have to be removed so that only the error due to the discretization of the velocity remains.

\subsection{Expected value of discretization errors}

The expected value $\mathcal{E}\left\{e_{\mathrm{d}}\right\}$ of the discretization errors is an important measure to study this random variable. By definition,

$\mathcal{E}\left\{e_{\mathrm{d}}\right\}=\frac{1}{Q} \sum_{q=1}^{Q} \iiint \int_{-\infty}^{\infty} e_{\mathrm{d} q} f_{U_{1}, \ldots, U_{4}}\left(u_{1}, \ldots, u_{4}\right) \mathrm{d} u_{1} \ldots \mathrm{d} u_{4}$

where $q=1,2, \ldots, Q$ runs over the grid of the discretized solution domain and

$e_{\mathrm{d} q} \approx-j 2 \pi f_{\mathrm{c}} \Delta \tau_{q} \exp \left\{-j 2 \pi f_{\mathrm{c}} \tau_{q}\right\}$

is the discretization errors at the $q$ th cell. If the $u_{m} \mathrm{~s}$ are statistically independent, the joint probability density function (PDF) is

$$
\begin{aligned}
& f_{U_{1}, \ldots, U_{4}}\left(u_{1}, \ldots, u_{4}\right)=f_{U_{1}}\left(u_{1}\right) \ldots f_{U_{4}}\left(u_{4}\right) \\
& \quad= \begin{cases}1 / 16, & -\mathbf{1} \preceq\left[u_{1}, \ldots, u_{4}\right]^{\mathrm{T}} \preceq \mathbf{1} \\
0, & \text { otherwise }\end{cases}
\end{aligned}
$$

where the symbol $\preceq$ means component-wise less than or equal. Combining Eqs. (22) and (28) with Eq. (26) results in

$\mathcal{E}\left\{e_{\mathrm{d}}\right\}=\frac{-j \pi f_{\mathrm{c}}}{8 c_{0} Q L} \sum_{i=1}^{L} \sum_{q=1}^{Q}\left(\exp \left\{-j 2 \pi f_{\mathrm{c}} \tau_{q}\right\} \iiint \int_{-1}^{1} \Delta \tau_{q} \mathrm{~d} u_{1} \ldots \mathrm{d} u_{4}\right)$.

In order to be able to find a feasible solution, $\Delta \tau_{q}$ is assumed to be an odd function of $u_{1}, u_{2}, u_{3}$ and $u_{4}$, the integration of this function between symmetric boundaries is equal to zero. So, it is derived that

$\mathcal{E}\left\{e_{\mathrm{d}}\right\}=0$.

This is an expected result and is also verified with the simulations.

\subsection{Variance of discretization errors}

By definition, the variance of the discretization errors is

$\mathcal{V}\left\{e_{\mathrm{d}}\right\}=\mathcal{E}\left\{\left(e_{\mathrm{d}}-\mathcal{E}\left\{e_{\mathrm{d}}\right\}\right)\left(e_{\mathrm{d}}-\mathcal{E}\left\{e_{\mathrm{d}}\right\}\right)^{*}\right\}$,

where the asterisk indicates complex conjugates. By inserting $\mathcal{E}\left\{e_{\mathrm{d}}\right\}$ from Eq. (30) into Eq. (31), it becomes

$\mathcal{V}\left\{e_{\mathrm{d}}\right\}=\mathcal{E}\left\{e_{\mathrm{d}} e_{\mathrm{d}}^{*}\right\}=4 \pi^{2} f_{\mathrm{c}}^{2} \mathcal{E}\left\{(\Delta \tau)^{2}\right\}$.

Hence,

$$
\begin{gathered}
\mathcal{V}\left\{e_{\mathrm{d}}\right\}=\frac{16 \pi^{2} f_{\mathrm{c}}^{2}}{c_{0}^{2} Q L} \sum_{i=1}^{L} \sum_{q=1}^{Q} \iiint \int_{-\infty}^{\infty}\left(\frac{\left(\boldsymbol{r}^{\mathrm{opt}}-\boldsymbol{r}^{\mathrm{sen}}\right) \cdot \Delta \boldsymbol{r}}{\left|\boldsymbol{r}^{\mathrm{opt}}-\boldsymbol{r}^{\mathrm{sen}}\right|}\right)^{2} \\
f_{U_{1}, \ldots, U_{4}}\left(u_{1}, \ldots, u_{4}\right) \mathrm{d} u_{1} \ldots \mathrm{d} u_{4} .
\end{gathered}
$$

Having $\left(\boldsymbol{r}^{\text {opt }}-\boldsymbol{r}^{\mathrm{sen}}\right)_{x}$ and $\left(\boldsymbol{r}^{\mathrm{opt}}-\boldsymbol{r}^{\mathrm{sen}}\right)_{y}$, respectively, the $x$ and $y$ components of $\left(\boldsymbol{r}^{\text {opt }}-\boldsymbol{r}^{\mathrm{sen}}\right)$, Eq. (33) becomes

$$
\begin{aligned}
& \mathcal{V}\left\{e_{\mathrm{d}}\right\}=\frac{4 \pi^{2} f_{\mathrm{c}}^{2}}{4 c_{0}^{2} Q L} \sum_{i=1}^{L} \sum_{q=1}^{Q} \iiint \int_{-1}^{1} \\
& \frac{\left(\left(\boldsymbol{r}^{\mathrm{opt}}-\boldsymbol{r}^{\mathrm{sen}}\right)_{x}\left(u_{1} \Delta r_{x}+u_{3} i T \Delta v_{x}\right)+\left(\boldsymbol{r}^{\mathrm{opt}}-\boldsymbol{r}^{\mathrm{sen}}\right)_{y}\left(u_{2} \Delta r_{y}+u_{4} i T \Delta v_{y}\right)\right)^{2}}{\left|\boldsymbol{r}^{\mathrm{opt}}-\boldsymbol{r}^{\mathrm{sen}}\right|^{2}} \\
& \quad \times f_{U_{1}, \ldots, U_{4}}\left(u_{1}, \ldots, u_{4}\right) \mathrm{d} u_{1} \ldots \mathrm{d} u_{4} .
\end{aligned}
$$

For the calculation of the variance, it is important to note that

$\int_{-1}^{1} \int_{-1}^{1} u_{i} u_{j} \mathrm{~d} u_{i} \mathrm{~d} u_{j}= \begin{cases}0 & i \neq j \\ 2 / 3 & i=j .\end{cases}$

Therefore, the variance in Eq. (35) transforms into

$$
\begin{aligned}
& \mathcal{V}\left\{e_{\mathrm{d}}\right\}=\frac{16 \pi^{2} f_{\mathrm{c}}^{2}}{9 c_{0}^{2} Q L} \sum_{i=1}^{L} \sum_{q=1}^{Q} \\
& \qquad \frac{\left(\boldsymbol{r}^{\mathrm{opt}}-\boldsymbol{r}^{\mathrm{sen}}\right)_{x}^{2}\left(\Delta r_{x}^{2}+i^{2} T^{2} \Delta v_{x}^{2}\right)+\left(\boldsymbol{r}^{\mathrm{opt}}-\boldsymbol{r}^{\mathrm{sen}}\right)_{y}^{2}\left(\Delta r_{y}^{2}+i^{2} T^{2} \Delta v_{y}^{2}\right)}{\left|\boldsymbol{r}^{\mathrm{opt}}-\boldsymbol{r}^{\mathrm{sen}}\right|^{2}} .
\end{aligned}
$$

Assuming uniform discretization steps (Fig. 2), i.e., $\Delta r_{x}=\Delta r_{y}=\Delta r_{x y}$ and $\Delta v_{x}=\Delta v_{y}=\Delta v_{x y}$ the variance in Eq. (35) simplifies into

$$
\begin{aligned}
\mathcal{V}\left\{e_{\mathrm{d}}\right\} & =\frac{16 \pi^{2} f_{\mathrm{c}}^{2}}{9 c_{0}^{2} Q L} \sum_{i=1}^{L} \sum_{q=1}^{Q}\left(\Delta r_{x y}^{2}+i^{2} T^{2} \Delta v_{x y}^{2}\right) \\
& =\frac{16 \pi^{2} f_{\mathrm{c}}^{2}}{9 c_{0}^{2}}\left(\Delta r_{x y}^{2}+\frac{1}{6}\left(2 L^{2}+3 L+1\right) T^{2} \Delta v_{x y}^{2}\right)
\end{aligned}
$$

The variance calculated in Eq. (36) is reduced by a grid refinement in position or velocity, as expected. Furthermore, the variance reduces by a lower carrier frequency or a shorter sampling time, theoretically. However, in many practical applications, this is not possible due to the system requirements and dimensions. With a larger carrier frequency, the spatial gird cells become larger compared to the wavelength. 


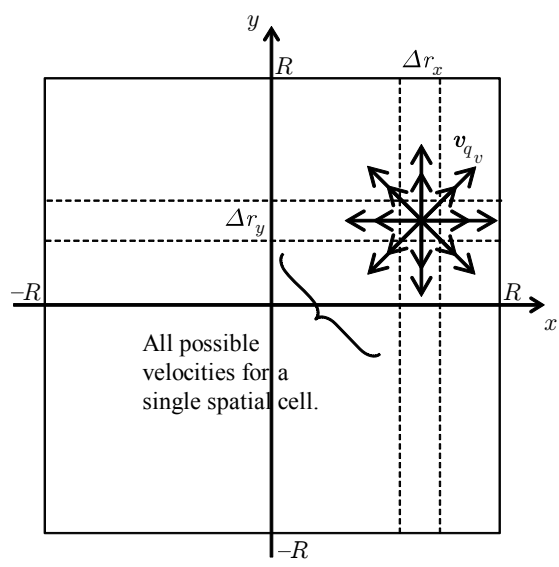

Figure 2. Discretization in $x$ and $y$. The discretization in velocity is shown by vectors from the center of the spatial grid cell.

Table 1. Definitions and values of the variables used for empirical assessments.

\begin{tabular}{lrll}
\hline Variable & Value & Unit & Description \\
\hline$T$ & 4 & $\mathrm{~ms}$ & sampling time \\
$f_{\mathrm{c}}$ & 24 & $\mathrm{GHz}$ & carrier frequency \\
$x_{\min }$ & -1 & $\mathrm{~m}$ & lower bound of $x$ \\
$x_{\max }$ & 1 & $\mathrm{~m}$ & upper bound of $x$ \\
$y_{\min }$ & -1 & $\mathrm{~m}$ & lower bound of $y$ \\
$y_{\max }$ & 1 & $\mathrm{~m}$ & upper bound of $y$ \\
$v_{\min }$ & -0.25 & $\mathrm{~m} \mathrm{~s}^{-1}$ & lower bound of $v$ \\
$v_{\max }$ & 0.25 & $\mathrm{~m} \mathrm{~s}^{-1}$ & upper bound of $v$ \\
\hline
\end{tabular}

Hence, the deviation from the centers of the cells, from which the discretization errors are computed, becomes larger. For a Doppler-only configuration, as of interest in this paper, the size of the grid cells is typically larger than the wavelength and, thus, the initial phase difference due to the position, according to the term with $\Delta r_{x y}^{2}$ in Eq. (36), would lead to very larger errors, which are not in agreement with the utilized assumption anymore. Therefore, the $\Delta r_{x y}^{2}$ term is not considered in our later evaluation of the error. In a practical radar application, the initial phase difference can be determined without discretization error, if an appropriate signal processing procedure is adopted.

\section{Empirical results of discretization errors}

Apart from the analytical solution, a possible approach for the characterization of the discretization errors is an empirical study. This can be a substitute method for applications in which finding the analytical solution is not straightforward.

In the empirical method, the difference between the best (or optimum) solution of the compressed sensing and the received signal is found by executing a large number of random constellations in a Monte Carlo experiment. It is important to

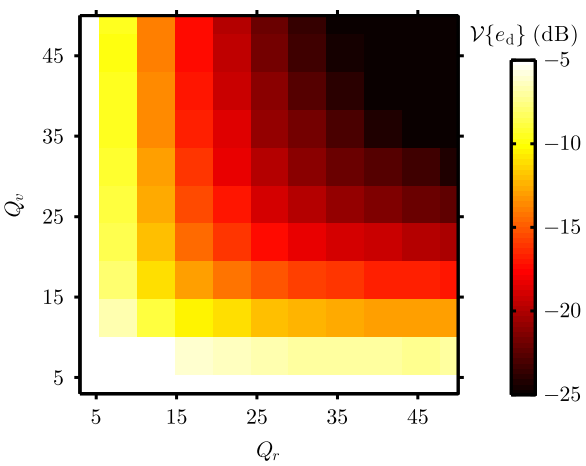

Figure 3. Variance of the discretization errors empirically computed for $5 \times 10^{5}$ targets in every constellation. The amplitudes of the received signals from the targets are assumed to be one. The variance is normalized to this amplitude and is reported in $\mathrm{dB}$. The number of steps in $r_{x}$ and $r_{y}$ and number of steps in $v_{x}$ and $v_{y}$ are $Q_{r}$ and $Q_{v}$, respectively, i.e., $Q_{r_{x}}=Q_{r_{y}}=Q_{r}$ and $Q_{v_{x}}=Q_{v_{y}}=Q_{v}$.

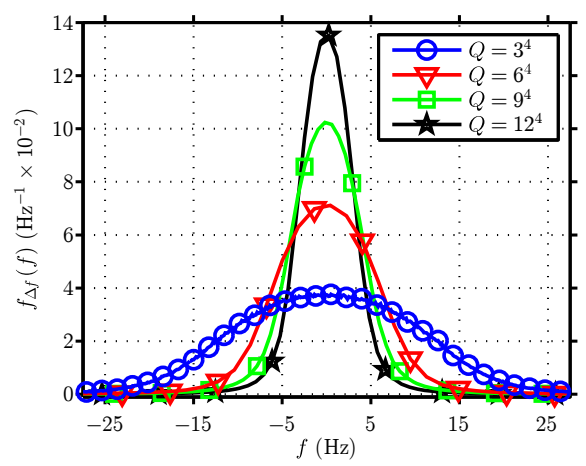

Figure 4. PDF of $\Delta f_{\mathrm{D}}$ computed for 4 scenarios. In this graph, $Q$ indicates the total number of grid cells. For example, $Q=3^{4}$ means there are 3 steps in each parameter and, hence, in total 81 cells exist.

note that the characterization of the discretization errors is no more a radar problem. Thus, for a given target, it is possible and also required to know the optimum solution as a priori without running any reconstruction algorithm.

Simulations for this purpose are set up based on the data of Table 1 . Then, a target with an arbitrarily chosen initial state, i.e., position and velocity, is considered in the scene. The initial state is in accordance with the solution domain limits. Based on the considered state, there is one cell among all grid cells which describes the state of the target the best. This is the reference cell for finding the discretization errors. This experiment is carried out for a large number of target states so that the discretization errors can be studied statistically.

Figure 3 illustrates the result of this experiment. For each constellation, $5 \times 10^{5}$ targets are simulated. There are 4 sensors placed based on a uniform distribution in the scene. The targets which are located in the very close vicinity of the sensors cause unexpected errors. Therefore, these targets are 
not considered in the simulation results. As expected, the results show that the discretization errors will be reduced by decreasing the discretization steps.

The discretization of the velocity leads to a difference between target's true Doppler and its estimation, considered as $\Delta f_{\mathrm{D}}=f_{\mathrm{D}}^{\mathrm{tar}}-f_{\mathrm{D}}^{\mathrm{opt}}$, in which $f_{\mathrm{D}}^{\mathrm{tar}}$ and $f_{\mathrm{D}}^{\mathrm{opt}}$ are the target's true Doppler and the estimated Doppler, respectively. Having the same configuration as above, in a further experiment, the PDF of $\Delta f_{\mathrm{D}}$ is estimated for 4 scenarios (Fig. 4). These scenarios vary with respect to the number of steps chosen to discretize the parameters. For each scenario, 5 sensors are used to find the difference between the Doppler frequency created by the $7.5 \times 10^{5}$ targets in the scene and the estimated ones. As shown in this figure, the average is very close to zero, which verifies the derivation in Eq. (30). The variance is also decreasing by increasing the number of discretization steps.

\section{Conclusion}

The discretization errors have been studied in this work as a separate source of impairments in an ideal Compressed Sensing problem. These errors, which occur due to discretization of the solution domain, are a result of modeling and cannot be suppressed by improving system attributes such as the input power or the system gain. Also, in a noise-less system, where measurements are carried out with infinite precision, these errors are still existing. The study showed that recovery without considering these errors leads to false or improper results. Including discretization errors helps to reduce the number of the iterations as well as the complexity of the recovery process. The recovery robustness increases by including these errors. The error was characterized analytically by taking the difference between the optimum solution derived by compressed sensing reconstruction algorithm and uniformly distributed targets in the solution domain. Furthermore, empirical results of the discretization errors obtained from a large number of simulations have been presented.

Data availability. The underlying research data can be requested from the authors.

Competing interests. The authors declare that they have no conflict of interest.
This work was supported by the German Research Foundation (DFG) and the Technische Universität München within the funding programme Open Access Publishing.

Edited by: Jens Anders

Reviewed by: two anonymous referees

\section{References}

Anitori, L., Maleki, A., Otten, M., Baraniuk, R., and Hoogeboom, P.: Design and Analysis of Compressed Sensing Radar Detectors, IEEE T. Signal Proces., 61, 813-827, 2013.

Azodi, H., Siart, U., and Eibert, T.: A Fast Three-Dimensional Deterministic Ray Tracing Coverage Simulator for a $24 \mathrm{GHz}$ AntiCollision Radar, Adv. Radio Sci., 11, 55-60, 2013.

Azodi, H., Wächter, T., Siart, U., Eibert, T., and Bonerz, S.: Compressed Sensing Approach for Fast Collision Warning Radar, in: Proceedings of German Microwave Conference (GeMiC), Aachen, Germany, 2014.

Azodi, H., Siart, U., and Eibert, T.: A Fast 3-D Deterministic Ray Tracing Coverage Simulator Including Creeping Rays Based On Geometry Voxelization Technique, IEEE T. Antenn. Propag., 63, 210-220, 2015.

Baraniuk, R.: Compressive Sensing [Lecture Notes], IEEE Signal Processing Magagazine, 24, 118-121, 2007.

Candès, E.: Compressive Sampling, Proceedings of the International Congress of Mathematicians, 1433-1452, Madrid, Spain, 2006.

Candes, E., Romberg, J., and Tao, T.: Stable Signal Recovery From Incomplete and Inaccurate Measurements, Commun. Pur. Appl. Math., 59, 1207-1223, 2006.

Chen, S., Donoho, D., and Saunders, M.: Atomic Decomposition by Basis Pursuit, SIAM review, 43, 129-159, 2001.

Donoho, D.: Compressed Sensing, IEEE T. Inform. Theory, 52, 1289-1306, 2006.

Donoho, D. and Tanner, J.: Precise Undersampling Theorems, Proceedings of IEEE, 98, 913-924, 2010.

Duarte, M. and Eldar, Y.: Structured Compressed Sensing: From Theory to Applications, IEEE T. Signal Proces., 59, 4053-4085, 2011.

Eldar, Y. and Kutyniok, G.: Compressed Sensing: Theory and Applications, Cambridge University Press, Cambridge, UK, 2012.

Eldar, Y., Kuppinger, P., and Bolcskei, H.: Block-Sparse Signals: Uncertainty Relations and Efficient Recovery, IEEE T. Signal Proces., 58, 3042-3054, 2010.

Ender, J.: A Brief Review of Compressive Sensing Applied to Radar, in: Proceedings of 14th International Radar Symposium (IRS), Vol. 1, 3-22, Dresden, Germany, 2013.

Fishler, E., Haimovich, A., Blum, R., Cimini Jr, L., Chizhik, D., and Valenzuela, R.: Spatial Diversity in Radars-Models and Detection Performance, IEEE T. Signal Proces., 54, 823-838, 2006.

Foucart, S. and Rauhut, H.: A Mathematical Introduction to Compressive Sensing, Birkhäuser, Basel, Switzerland, 2013.

Gurbuz, A., Teke, O., and Arikan, O.: Sparse Ground-Penetrating Radar Imaging Method for Off-the-Grid Target Problem, J. Electron. Imaging, 22, 1-8, 2013. 
Haimovich, A., Blum, R., and Cimini, L.: MIMO Radar With Widely Separated Antennas, IEEE Signal Processing Magazine, 25, 116-129, 2008.

Herman, M. and Strohmer, T.: High-Resolution Radar Via Compressed Sensing, IEEE T. Signal Proces., 57, 2275-2284, 2009.

Hurst, M. and Mittra, R.: Scattering Center Analysis Via Prony's Method, IEEE T. Antenn. Propag., 35, 986-988, 1987.

Lehmann, N., Haimovich, A., Blum, R., and Cimini, L.: High Resolution Capabilities of MIMO Radar, in: Proceedings of 40th Asilomar Conference on Signals, Systems and Computers (ACSSC), 25-30, IEEE, 2006.

Nielsen, J. K., Christensen, M. G., and Jensen, S. H.: On compressed sensing and the estimation of continuous parameters from noisy observations, in proceedings of 2012 IEEE International Conference on Acoustics, Speech and Signal Processing (ICASSP), 3609-3612, Kyoto, IEEE, 2012.
Skolnik, M.: Introduction to Radar Systems, McGraw-Hill, New York City, NY, USA, 1962.

Tan, Z. and Nehorai, A.: Sparse Direction of Arrival Estimation Using Co-Prime Arrays With Off-Grid Targets, IEEE T. Signal Proces., 21, 26-29, 2014.

Tang, G., Bhaskar, B., Shah, P., and Recht, B.: Compressive Sensing Off the Grid, in: Proceedings of 50th Annual Allerton Conference on Communication, Control and Computing, 778-785, Monticello, IL, USA, 2012.

Wächter, T., Siart, U., Eibert, T., and Bonerz, S.: Multi-Sensor Doppler Radar for Machine Tool Collision Detection, Adv. Radio Sci., 12, 35-41, 2014. 